Original Research Paper

\title{
The Construction of Two and Three Dimensional Molecular Models for the miR-31 and Its Silencer as the Triple Negative Breast Cancer Biomarkers
}

\author{
Arli Aditya Parikesit \\ Department of Bioinformatics, School of Life Sciences, \\ Indonesia International Institute for Life Sciences, Jakarta, Indonesia
}

\author{
Article history \\ Received: 14-06-2018 \\ Revised: 22-06-2018 \\ Accepted: 16-10-2018 \\ Tel: +62 2129567888 \\ Email: arli.parikesit@i3l.ac.id
}

\begin{abstract}
The Triple Negative Breast Cancer (TNBC) is considered as the most difficult Breast Cancer type to be medicated. So far, the acceptable medication and diagnostics measure are based upon the proteomics-world view, especially with utilizing the natural products and their derivatives as drug candidates. However, as the transcriptomicsstudies are getting more advanced, the research on non-coding (nc)RNA is being considered as a more feasible approach to deal with the TNBC. The miR-31, as the ncRNA that play part in the molecular mechanism of the TNBC, is extensively studied mainly for providing the biomarker and drug candidate. However, the molecular interaction and structures are mainly unknown and it is subject of investigation of this research. This research is utilizing the interplay of pipelines that consist of the Vienna RNA package for 2D RNA structure prediction and the simRNA/modeRNA packages for 3D RNA structure prediction. The result is a blueprint that satifactionary illustrates the RNA structure in a fine-grained manner. The miR-31 and its respective silencing (si)RNA are indeed could be useful to be biomarkers and blue-print for the drug design of the TNBC.
\end{abstract}

Keywords: TNBC, ncRNA, miR-31, Transcriptomics, Drug Design

\section{Introduction}

Breast cancer, especially the Triple Negative subtype (TNBC), has been designated as one of the cause of death for women (WHO, 2016). The difficult challenge in dealing with the TNBC is its tendency to not responsive towards the endocrine-based therapy and other standard therapeutics agents (Hudis and Gianni, 2011). The diagnostics and medication for the TNBC still mainly focused on proteomics-based approach (Cleator et al., 2007; Lehmann et al., 2011; Widodo et al., 2014). However, as transcriptomics research is getting more advanced, this particular approach is currently under consideration as well to treat breast cancer. miRNA-451, as short strand non-coding RNA, has been found to act as a biomarker in breast cancer (Kovalchuk et al., 2008). Meanwhile, the miRNA-155 has been found as TNBC biomarker, as well as the interplay of the microRNAs that upregulated in the cells (Radojicic et al., 2011; Kong et al., 2014). The molecular mechanism of the TNBC was studied with the application of silencing
(si)RNA that blocks the expression of the drugresistance gene (Deng et al., 2013).

However, special attention should be given to the miR31 TNBC biomarker, as it has influence on the occurrence of the hypermethylation of its host gene, the LOC554202 promoter-associated CpG island (Augoff et al., 2012). In this respect, the miR-31 exerts extensive epigenetics properties as well. Moreover, the miR-31 also influences suppressor of the Wnt signaling antagonist to expand the breast tumorigenesis ( $\mathrm{Lv}$ et al., 2017). Special care is advised as the miR-31 also shows expression in the hormone-based breast cancer (Lu et al., 2012).

Based upon the molecular simulation method, the availability of the 2D and 3D model of drug candidates and biomarkers are very important to create a solid blue print of the drug design (Kinjo and Nishikawa, 2005; Arnold et al., 2006; Sripakdeevong et al., 2012). This theory could be applied for the transcriptomics-based drug design and biomarkers study as well. The file format that utilized for the 3D structure of RNA still uses the Protein Data Bank (PDB) one. Two 3D RNA databases are available; they are RCSB (rcsb.org) and 
NDB (http://ndbserver.rutgers.edu). The objective of this research is to predict the $2 \mathrm{D}$ and $3 \mathrm{D}$ model of miR-31 and its respective siRNA.

\section{Material and Methods}

\section{Material}

The established pipelines were utilized to construct the 2D and 3D models of the RNAs (Parikesit et al., 2016; Parikesit and Anurogo, 2016; 2018; Parikesit and Nurdiansyah, 2018). Both the 2D and 3D model pipelines were employed to ensure the vivid resolution of the graphics. The utilized software and hardware were the standard Windows 10 based-notebook with a broadband Internet connection.

\section{Sequence Alignment}

The primary genome database of NCBI was employed in in https://www.ncbi.nlm.nih.gov/, in order to search for the miR-31 sequences. The representative samples from the mammalian kingdoms were taken for comparative study, although the main focus would be on the Human (Homo sapiens) data. The FASTA format file were downloaded and curated with notepad ++ text editor (https://notepad-plus-plus.org).

\section{Two-Dimensional Modeling}

The 2D structure and interactions data between the miR-31 and siRNA were generated with the Vienna RNA Package with the online version in here: http://rna.tbi.univie.ac.at/ (Gruber et al., 2015). The FASTA-based sequences were provided as input to the web application (TBI, 2016). Computation process was conducted only with the default parameters. These respective Vienna RNA Package-based applications will be utilized for generating the 2D data: RNAfold (Gruber et al., 2008; Lorenz et al., 2011), RNAalifold (Hofacker et al., 2002; Bernhart et al., 2008; Gruber et al., 2008), RNAup (Muckstein et al., 2006; Gruber et al., 2008), Barriers (Flamm et al., 2002; Wolfinger et al., 2004) and RNAxs (Tafer et al., 2008).

The inhibition of the miR-31 and its respective siRNA will be computed with those tools based on the biochemical thermodynamics and kinetics properties. In order to annotate the functional features of the miR-31 biomarkers in the cell, the $2 \mathrm{D}$ and $3 \mathrm{D}$ structural determination is essential. The Vienna format data files are the outputs of those procedures (Smit et al., 2008).

\section{Three-Dimensional Modeling}

The software of both simRNA (https://genesilico.pl/SimRNAweb/) and modeRNA (http://iimcb.genesilico.pl/modernaserver/) were forwarded with the $2 \mathrm{D}$ data in order to annotate the respective 3D structure (Rother et al., 2011; Magnus et al., 2016). The Protein Data Bank (PDB) files were created for further visualization and analysis.

\section{Data Analysis}

The 2D and 3D files were visualized to observe their structural conservation. Additional information from the PDB website (https://www.rcsb.org) was employed to interpret the function of the RNA in the cells.

\section{Results}

The following miR-31 sequences in Table 1 were found in the Genbank/NCBI database. They consisted of the 9 different mammals, from the different families as well. Those sequences were retrieved for the comparative studies within this research, especially for the functional and structural annotations of the RNA.

\section{RNAalifold Conserved Structural Alignment}

In order to comprehend the structural variations of miR-31 within the mammalian kingdom, RNAalifold was employed to search for the 2D structural conservation. Hence, the structure was elucidated in Fig. 1a. In this respect, the color of the annotations clearly shows that the structure was highly conserved in the mammalian kingdom. It could be seen in the 2D structure of human miR-31, which is closely resembles the conserved structure (Fig. 1b). This homology is clearly a sign that the miR-31 2D structure of the mammalian kingdom in general was derived from a single structure, as if that is not the case, the conserved structure could not be able to be generated accordingly.

Table 1: The miR-31 genes in Genbank/NCBI database

\begin{tabular}{lll}
\hline No. & Accession code & Description \\
\hline 1. & gi|387763215|ref|NR_049716.1| & Bos taurus microRNA 31 (MIR31), microRNA \\
2. & gi|270132979|ref|NR_032004.1| & Pan troglodytes microRNA 31 (MIR31), microRNA \\
3. & gi|731442466|ref|NR_049225.1| & Canis lupus familiaris microRNA 31 (MIR31), microRNA \\
4. & gi|270132836|ref|NR_032994.1| & Equus caballus microRNA 31 (MIR31), microRNA \\
5. & gi $|262205650|$ ref|NR_029747.1| & Mus musculus microRNA 31 (Mir31), microRNA \\
6. & gi $|262205714|$ ref|NR_029505.1| & Homo sapiens microRNA 31 (MIR31), microRNA \\
7. & gi|296923669|ref|NR_031845.1| & Rattus norvegicus microRNA 31 (Mir31), microRNA \\
8. & gi|731441896|ref|NR_128414.1| & Sus scrofa microRNA 31 (MIR31), microRNA \\
9. & gi|563318615|ref|NR_106227.1| & Gorilla gorilla microRNA 31 (MIR31), microRNA \\
\hline
\end{tabular}




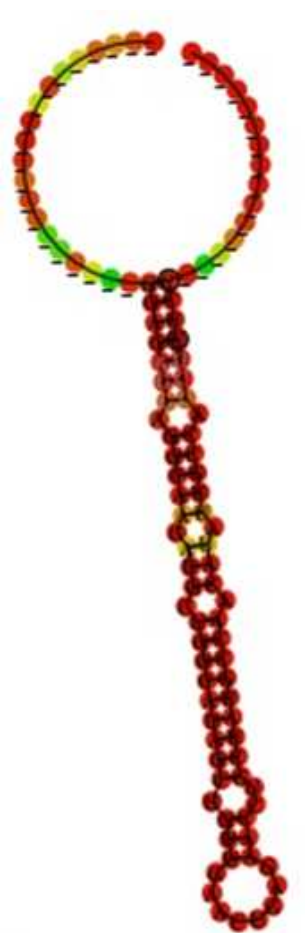

(a)

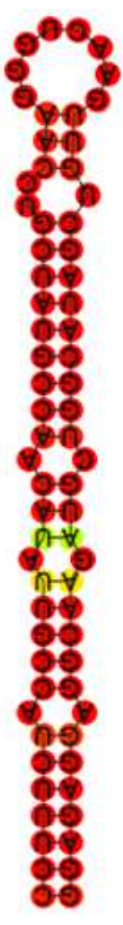

(b)

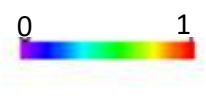

(c)

Fig. 1: Structure of miR-31: (a) RNAlifold conserved structure (b) RNAfold Homo sapiens (c) The scale of base pair probability, 0 refers to the improbable structure and/or sequence, while 1 refers to the most probable ones.

Based on the RNAalifold calculation, the conserved $2 \mathrm{D}$ structure is having minimum predicted free energy of - $53.28 \mathrm{kcal} / \mathrm{mol}$. While the $2 \mathrm{D}$ structure of miR-31 Homo sapiens is $-36.80 \mathrm{kcal} / \mathrm{mol}$. Both structures provided spontaneous values and this is a strong indication that those structures indeed exist. However, the half time of those chemical species could be very short, in the fraction of nanoseconds.

\section{Barriers Server}

The biochemical reaction transition could only be able to visualize clearly in a computational environment. The barriers application was utilized to predict the trajectory of the RNA molecules in a fine-grained manner. In this respect, the transition state of the human miR-31 molecule was exposed in Fig. 2. Surprisingly, the barrier server predicted 10,082,079 structures in an energy range of $30.2 \mathrm{kcal} / \mathrm{mol}$ above the minimum free energy. The extreme diversity of the predicted transition structure is a clear indication of the molecular dynamism of the molecule and a sign of its possible variety of the functional features.

\section{siRNA Prediction}

As they are various theories that exist concerning the role of the miR-31 in the breast cancer, one feasible way is to generate siRNA to prevent its overexpression. The RNAxs application was utilized to create the siRNA that interacted with the miR-31 of the human (Fig. 3). Thus, based upon the execution of the RNAup program, the interaction was shown to be feasible because of the spontaneous reaction with free energy value of -24.94 $\mathrm{kcal} / \mathrm{mol}$.

\section{D Model of the RNA}

Based on the homology search on the modeRNA database, it was found that the miR-31 is strikingly homologous with the tRNA from Pyrococcus horikoshii, an arachea that isolated from the hydrothermal vent in Okinawa, Japan (Fig. 4). It is interesting to see that the tRNA is in a complex with the arginyl-tRNA synthetase protein. It is a one example that Protein-RNA complex indeed exist in the arachea cell. The computed data is in the PDB format file. The 3D homology model of the siRNA miR-31 was not found in the database. However, it was found that it belongs to the RNA family of RF00275 in RFAM database. This family acts as small nucleolar (sno)RNA U56, which is a ncRNA that play part in snRNA biogenesis (http://rfam.xfam.org/family/RF00275).

Both Jena3D (http://jenalib.leibniz-fli.de/) and UCSF Chimera were utilized to visualize the de novo model of the miR-31 and siRNA mir 31 (Fig. 5). Both visualization packages were chosen due to their compatibility with the nucleic acid 3D structure file of PDB format. The de novo models did not show any binding with other proteins because the data input only restricted to the RNA. 


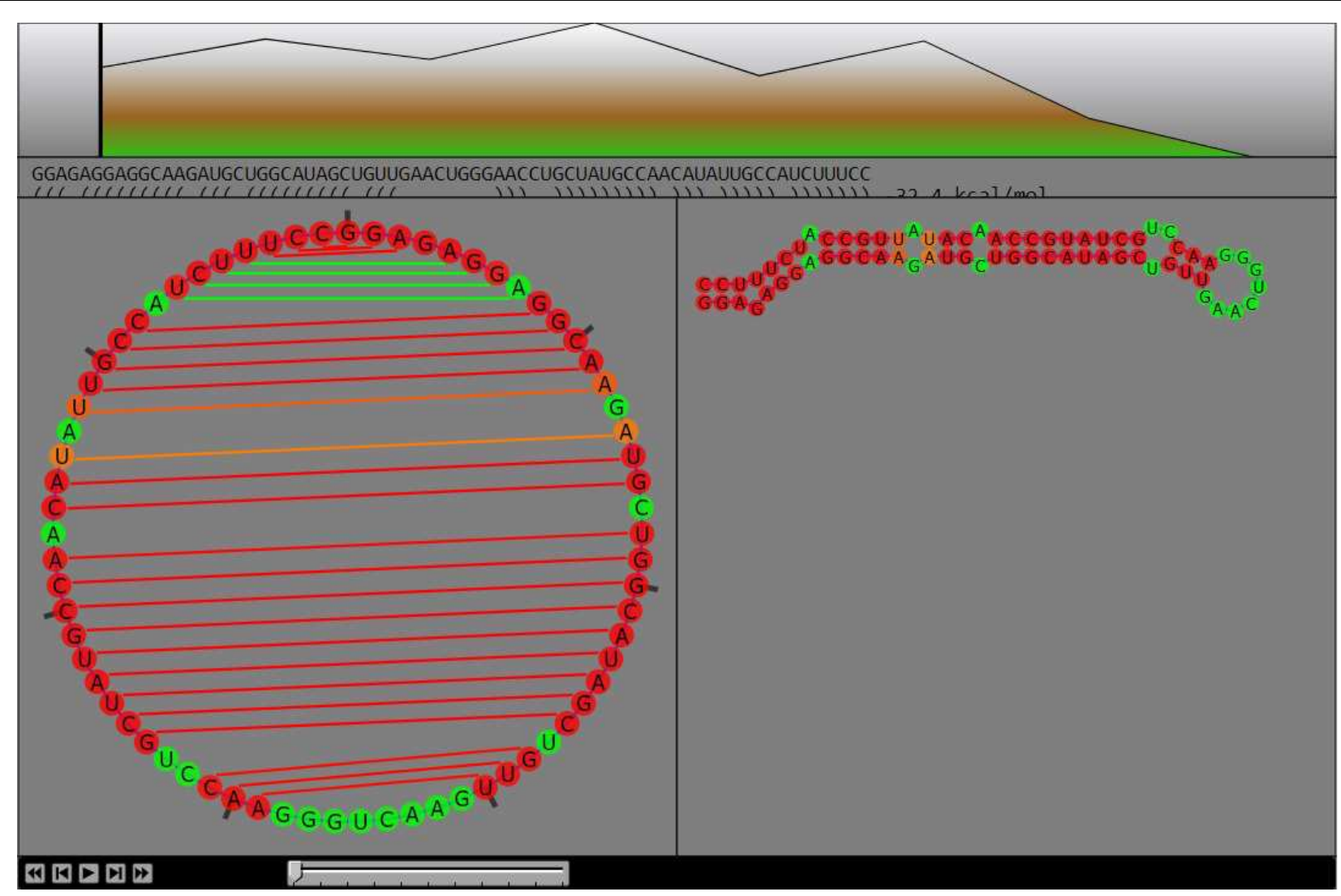

Fig. 2: Barrier server of the miR-31 Homo sapiens. The picture above shows the probability of forming the structure according to the animation sequence. The left circle graph is the graph theory representation of each DNA sequence. The right-hand structure shows the formation prediction structure, with its probability at each sequence

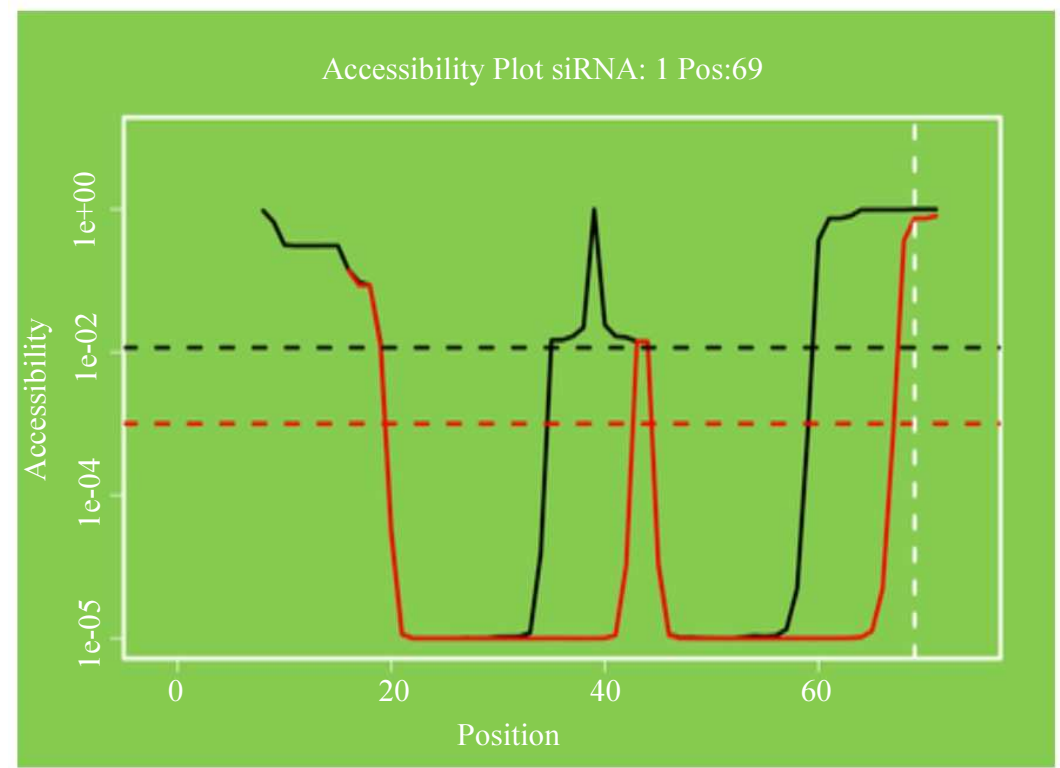

\begin{tabular}{|l|r|}
\hline siRNA & 1 \\
\hline Worst Rank & 1 \\
\hline Position & 69 \\
\hline Access 8nt & 0.9919 \\
\hline Access 16nt & 0.7379 \\
\hline Assymetry (S) & 1.0000 \\
\hline Assymetry (E) & 0.8448 \\
\hline Self Folding & 1.0000 \\
\hline Free End & 1.0000 \\
\hline Target Seq. & CCAACAUAUUGCCAUCUUU \\
\hline siRNA Seq. & AAAGATGGCAATATGTTGG \\
\hline BLAST & NCBI BLAST \\
\hline
\end{tabular}

Fig. 3: The RNAxs output of the miR-31 siRNA. The black plot refers to the accessibility of the target sequence and the red plot refers to the accessibility of siRNA sequence. If approaching zero or touching the base of X-axis, the probability of accessibility for the sequence is zero. If it farther from zero, the probability will be higher 


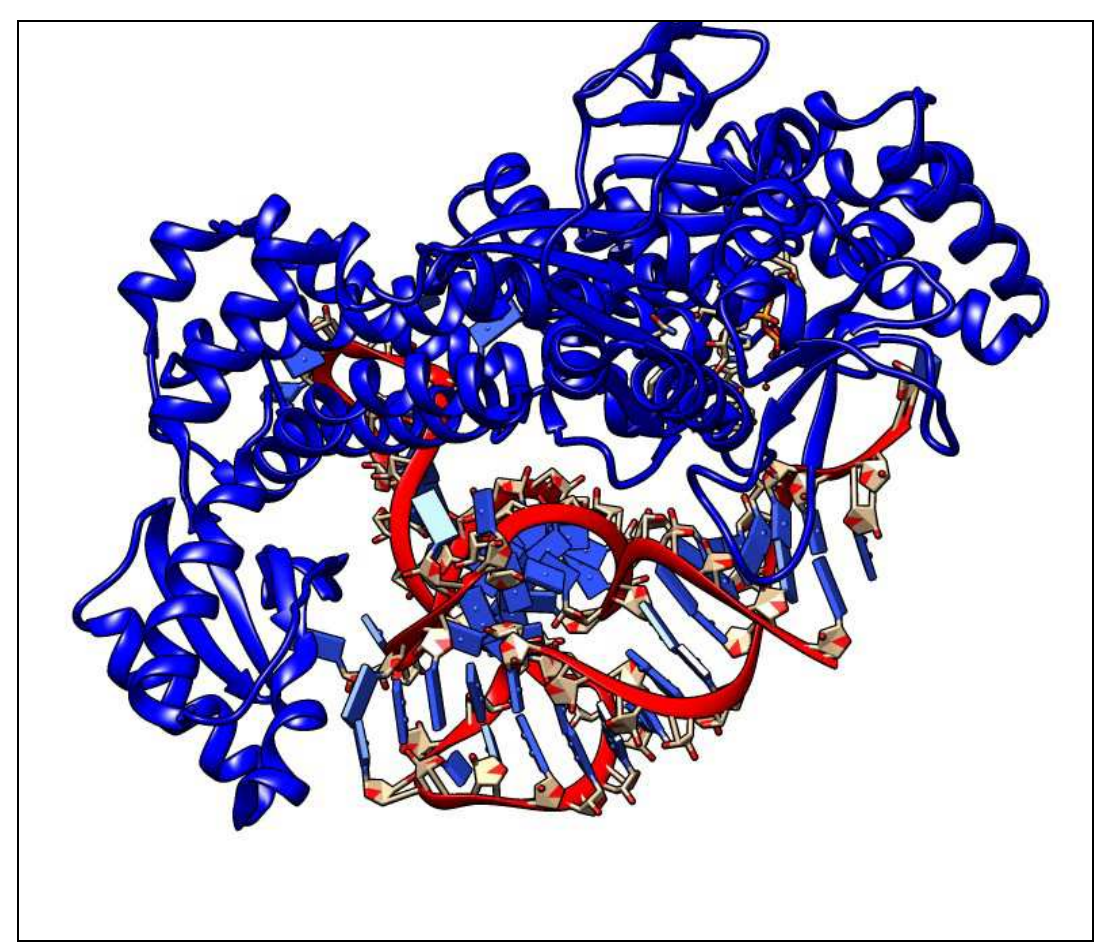

Fig. 4: The homology model of the miR-31 with modeRNA software. Crystal structure of Pyrococcus horikoshii arginyl-tRNA synthetase complexed with tRNA(Arg) and an ATP analog (ANP) (PDB ID: 2ZUE, RFAM ID: RF00005)

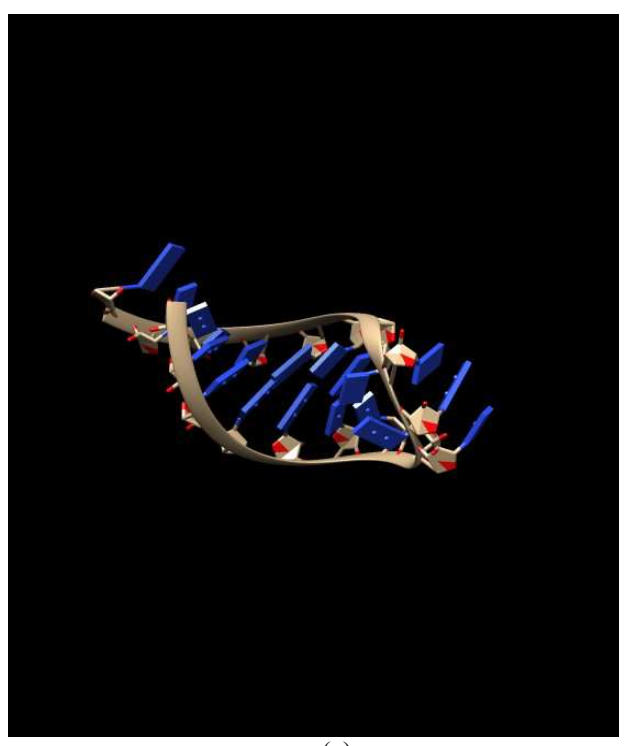

(a)

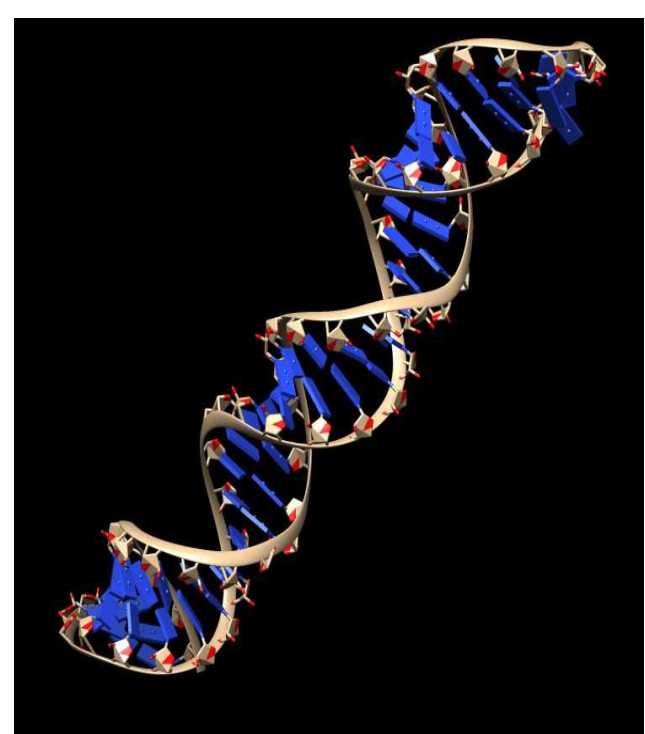

(b)

Fig. 5: Visualization by UCSF Chimera for de novo model of a) miR-31 b) siRNA miR-31

\section{Discussion}

The inability for the TNBC to provide significant response toward the hormonal therapy only left the viable option for diagnostics and medication in the transcriptomics realm. The Human Genome Project (HGP) that mainly constructed with the proteomics- world view, has ultimate goal to provide blue-print for the diagnostics, prevention and therapy for all kind of diseases, especially cancer as 'the emperor of all maladies' (Teufel et al., 2006). However, the completion of HGP still can't cope with the challenge that posed by the complexity of the molecular mechanism of diseases. The interplay of genes, proteins and RNAs play part in 
the progression of cancer. In this respect, it is very difficult to only inspect the aspect of disease with only one provided insight. In order to supplement the HGP, new database has been provided, namely the RNAcentral (RNAcC, 2017). It provides insight that missing in the proteomics study, mainly for TNBC that unable to be resolved with proteomics-based approach. The RNAcentral is integrated with the NCBI refseq in order to provide coherent continuation with the HGP initiative. This worldwide initiative is a manifestation of the importance of the transcriptomics research, especially in studying cancer.

As the set of tools that taking advantage of the RNAcentral database, Vienna RNA package provided solid approach for providing a fine-grained resolution of the RNA 2D structure (Gruber et al., 2008). The elucidation of the RNA 2D structure provided very difficult as the RNA doesn't have clearly-defined functional unit like protein-domain. The RNA 2D structure data in the 'vienna dot bracket' format was indeed useful for further elucidation pipeline of a 3D structure with the simRNA and modeRNA tools to provide the PDB output file. The provided PDB output is solid evidence that the molecular modeling of the TNBC biomarkers and drug candidate is indeed feasible.

The notable challenge is the impossibility of leaving behind the whole proteomics approach, especially for studying TNBC. As the non-coding RNAs is mainly an expression products of the proteins interplay, the proteinRNA interactions studies are getting more important (Murchison and Hannon, 2004; Gregory and Shiekhattar, 2005; Cheloufi et al., 2010). The modules and applications to inspect the networks and docking of ProteinRNA are developed extensively (Pérez-Cano et al., 2010; Eargle and Luthey-Schulten, 2012; Li et al., 2014). This particular approach is an interesting option to proceed for investigating the molecular mechanism of TNBC further in the future. Thus, it should be supported with the sufficient in-house GRID-based computational resources, as relying on the external web-based application is possibly insufficient due to the certain computational limitation (Heru Suhartanto, 2012; van Schaik et al., 2012; Jaber et al., 2013).

\section{Conclusion}

It was found that the $2 \mathrm{D}$ and $3 \mathrm{D}$ structures of the miR-31 and its siRNA could be elucidated with a finegrained resolution using the molecular modeling software and it could be useful as the TNBC biomarker and drug candidate. However, the influence of the protein molecular simulation is still very strong as the utilities were created based on the PDB format file. The future venue of this research would be utilizing deeper molecular simulation perspective.

\section{Acknowledgement}

The author would like to thanks the Research and Community Engagements Institute (LPPM) and the IT Department of i3L for their heartfelt supports

\section{Funding Information}

The author would like to thanks to Direktorat Riset dan Pengabdian Masyarakat, Direktorat Jenderal Penguatan Riset dan Pengembangan Kementerian Riset, Teknologi, dan Pendidikan Tinggi Republik Indonesia for providing Hibah Penelitian Berbasis Kompetensi DIKTI/KOPERTIS III 2018 No. 049/KM/PNT/2018

\section{Author's Contributions}

Arli Aditya Parikesit conducted the research and involved in the preparation, development and publication of this manuscript.

\section{Ethics}

The author declares that there is no competing interest with any party.

\section{References}

Arnold, K., L. Bordoli, J. Kopp and T. Schwede, 2006. The SWISS-MODEL workspace: A web-based environment for protein structure homology modelling. Bioinformatics, 22: 195-201.

DOI: $10.1093 /$ bioinformatics/bti770

Augoff, K., B. McCue, E.F. Plow and K. Sossey-Alaoui, 2012. MiR-31 and its host gene lncRNA LOC554202 are regulated by promoter hypermethylation in triple-negative breast cancer. Molecular Cancer, 11: 5-5.

DOI: $10.1186 / 1476-4598-11-5$

Bernhart, S.H., I.L. Hofacker, S. Will, A.R. Gruber and P.F. Stadler, 2008. RNAalifold: Improved consensus structure prediction for RNA alignments. BMC Bioinformat., 9: 474-474.

DOI: $10.1186 / 1471-2105-9-474$

Cheloufi, S., C.O. Dos Santos, M.M.W. Chong and G.J. Hannon, 2010. A dicer-independent miRNA biogenesis pathway that requires Ago catalysis. Nature, 465: 584-589. DOI: 10.1038/nature09092

Cleator, S., W. Heller and R.C. Coombes, 2007. Triplenegative breast cancer: Therapeutic options. Lancet Oncol., 8: 235-244.

DOI: $10.1016 / \mathrm{S} 1470-2045(07) 70074-8$

Deng, Z.J., S.W. Morton, E. Ben-Akiva, E.C. Dreaden and K.E. Shopsowitz et al., 2013. Layer-by-layer nanoparticles for systemic codelivery of an anticancer drug and siRNA for potential triplenegative breast cancer treatment. ACS Nano, 7: 9571-9584. DOI: $10.1021 / \mathrm{nn} 4047925$ 
Eargle, J. and Z. Luthey-Schulten, 2012. NetworkView: $3 \mathrm{D}$ display and analysis of protein \{middle dot\}RNA interaction networks. Bioinformatics, 28: 3000-3001. DOI: $10.1093 /$ bioinformatics/bts546

Flamm, C., I.L. Hofacker, P.F. Stadler, T. Wolfinger and M.T. Wolfinger, 2002. Barrier trees of degenerate landscapes. Zeitschrift für Physikalische Chemie, 216: 155-155. DOI: 10.1524/zpch.2002.216.2.155

Gregory, R.I. and R. Shiekhattar, 2005. MicroRNA biogenesis and cancer. Cancer Res., 65: 3509-3512. DOI: 10.1158/0008-5472.CAN-05-0298

Gruber, A.R., S.H. Bernhart and R. Lorenz, 2015. The ViennaRNA Web Services. In: RNA Bioinformatics, Picardi, E. (Ed.), Humana Press, New York, pp: 307-326.

Gruber, A.R., R. Lorenz, S.H. Bernhart, R. Neuböck and I.L. Hofacker, 2008. The Vienna RNA websuite. Nucleic Acids Res., 36: W70-W74.

DOI: $10.1093 / \mathrm{nar} / \mathrm{gkn} 188$

Heru Suhartanto, A.Y., 2012. Performance analysis cluster computing environments on molecular dynamic simulation of RAD GTPase and LOXCurcumin molecules with AMBER. Int. J. Comput. Sci., 9: 90-96.

Hofacker, I.L., M. Fekete and P.F. Stadler, 2002. Secondary structure prediction for aligned RNA sequences. J. Molecular Biol., 319: 1059-1066. DOI: $10.1016 / \mathrm{S} 0022-2836(02) 00308-\mathrm{X}$

Hudis, C.A. and L. Gianni, 2011. Triple-negative breast cancer: An unmet medical need. Oncologist, 16: 1-11. DOI: 10.1634/theoncologist.2011-S1-01

Jaber, A.S., M. Othman and M.F. Sjaugi, 2013. Grid computing and scientific research: Concepts and review. Proceedings of the International Conference on Research and Innovation in Information Systems, Nov. 27-28, IEEE Xplore Press, Kuala Lumpur, Malaysia, pp: 156-161.

DOI: 10.1109/ICRIIS.2013.6716701.

Kinjo, A.R. and K. Nishikawa, 2005. Recoverable onedimensional encoding of three-dimensional protein structures. Bioinformatics, 21: 2167-2170.

DOI: $10.1093 /$ bioinformatics/bti330

Kong, W., L. He, E.J. Richards, S. Challa and C.X. $\mathrm{Xu}$ et al., 2014. Upregulation of miRNA-155 promotes tumour angiogenesis by targeting VHL and is associated with poor prognosis and triplenegative breast cancer. Oncogene, 33: 679-689. DOI: $10.1038 /$ onc. 2012.636

Kovalchuk, O., J. Filkowski, J. Meservy, Y. Inytskyy and V.P. Tryndyak et al., 2008. Involvement of microRNA-451 in resistance of the MCF-7 breast cancer cells to chemotherapeutic drug doxorubicin. Molecular Cancer Therapeut., 7: 2152-2159.

DOI: $10.1158 / 1535-7163 . M C T-08-0021$
Lehmann, B.D., J.A. Bauer, X. Chen, M.E. Sanders and A.B. Chakravarthy et al., 2011. Identification of human triple-negative breast cancer subtypes and preclinical models for selection of targeted therapies. J. Clin. Invest., 121: 2750-2767. DOI: $10.1172 / \mathrm{JCI} 45014$

Li, J.H., S. Liu, H. Zhou, L.H. Qu and J.H. Yang, 2014. starBase v2.0: Decoding miRNA-ceRNA, miRNAncRNA and protein-RNA interaction networks from large-scale CLIP-Seq data. Nucleic Acids Res., 42: D92-D97. DOI: 10.1093/nar/gkt1248

Lorenz, R., S.H. Bernhart, C. zu Siederdissen, H. Tafer and C. Flamm et al., 2011. \{ViennaRNA\} Package 2.0. Algorithms Molecular Biol., 6: 26-26. DOI: 10.1186/1748-7188-6-26

Lu, Z., Y. Ye, D. Jiao, J. Qiao and S. Cui et al., 2012. miR-155 and miR-31 are differentially expressed in breast cancer patients and are correlated with the estrogen receptor and progesterone receptor status. Oncol. Lett., 4: 1027-1032. DOI: $10.3892 / \mathrm{ol} .2012 .841$

Lv, C., F. Li, X. Li, Y. Tian and Y. Zhang et al., 2017. MiR-31 promotes mammary stem cell expansion and breast tumorigenesis by suppressing Wnt signaling antagonists. Nat. Commun., 8: 1036-1036. DOI: $10.1038 / \mathrm{s} 41467-017-01059-5$

Magnus, M., M.J. Boniecki, W. Dawson and J.M. Bujnicki, 2016. SimRNAweb: A web server for RNA 3D structure modeling with optional restraints. Nucleic Acids Res., 44: gkw279-gkw279. DOI: $10.1093 / \mathrm{nar} / \mathrm{gkw} 279$

Muckstein, U., H. Tafer, J. Hackermuller, S.H. Bernhart and P.F. Stadler et al., 2006. Thermodynamics of RNA-RNA binding. Bioinformatics, 22: 1177-1182. DOI: $10.1093 /$ bioinformatics/btl024

Murchison, E.P. and G.J. Hannon, 2004. miRNAs on the move: miRNA biogenesis and the RNAi machinery. Curr. Opin. Cell Biol., 16: 223-229. DOI: $10.1016 /$ J.CEB.2004.04.003

Parikesit, A.A. and D. Anurogo, 2016. Prediksi struktur 2-dimensi non-coding RNA dari biomarker kanker payudara triple-negative dengan vienna RNA package. Chim. Natura Acta, 4: 27-27. DOI: $10.24198 /$ cna.v4.n1.10445

Parikesit, A.A. and D. Anurogo, 2018. 3D prediction of breast cancer biomarker from the expression pathway of lincrna-ror/Mir-145/Arf6. J. Sains Teknol., 2: 10-19.

Parikesit, A.A., D. Anurogo and A.A. Parikesit, 2016. Bioinformatics approach towards transcriptomics of filaggrin. J. Agromedicine Med. Sci., 2: 8-16.

Parikesit, A.A. and R. Nurdiansyah, 2018. Generating two-dimensional repertoire of siRNA linc-ROR and siRNA mRNA ARF6 from the lincRNA-RoR/miR145/ARF6 expression pathway that involved in the progression of triple negative breast cancer. Mater. Sci. Eng., 299: 012059-012059.

DOI: $10.1088 / 1757-899 X / 299 / 1 / 012059$ 
Pérez-Cano, L., A. Solernou, C. Pons and J. FernándezRecio, 2010. Structural prediction of protein-RNA interaction by computational docking with propensity-based statistical potentials. Pacific Symposium on Biocomputing. DOI: 9789814295291_0031

Radojicic, J., A. Zaravinos, T. Vrekoussis, M. Kafousi and D.A. Spandidos et al., 2011. MicroRNA expression analysis in triple-negative (ER, PR and Her2/neu) breast cancer. Cell Cycle, 10: 507-517. DOI: $10.4161 / \mathrm{cc} .10 .3 .14754$

Rother, M., K. Milanowska, T. Puton, J. Jeleniewicz and K. Rother et al., 2011. ModeRNA server: An online tool for modeling RNA 3D structures. Bioinformatics, 27: 2441-2442.

DOI: 10.1093/bioinformatics/btr400

van Schaik, B.D.C., M. Santcroos, V. Korkhov, A. Jongejan and M. Willemsen et al., 2012. Challenges in DNA sequence analysis on a production grid.

Smit, S., K. Rother, J. Heringa and R. Knight, 2008. From knotted to nested RNA structures: A variety of computational methods for pseudoknot removal. RNA, 14: 410-416. DOI: 10.1261/rna.881308

Sripakdeevong, P., K. Beauchamp and R. Das, 2012. RNA 3D structure analysis and prediction. RNA 3D Structure Anal. Predict., 27: 43-65. DOI: $10.1007 / 978-3-642-25740-7$
Tafer, H., S.L. Ameres, G. Obernosterer, C.A. Gebeshuber and R. Schroeder et al., 2008. The impact of target site accessibility on the design of effective siRNAs. Nat. Biotechnol., 26: 578-583. DOI: $10.1038 /$ nbt 1404

TBI, 2016. Vienna RNA package web version 2.0. http://rna.tbi.univie.ac.at/\#intro

Teufel, A., M. Krupp, A. Weinmann and P.R. Galle, 2006. Current bioinformatics tools in genomic biomedical research (Review). Int. J. Molecular Med., 17: 967-973.

RNAcC, 2017. RNAcentral: A comprehensive database of non-coding RNA sequences. Nucleic Acids Res., 45: D128-D134. DOI: 10.1093/nar/gkw1008

WHO, 2016. WHO breast cancer prevention and control. WHO.

Widodo, I., E.K. Dwianingsih, E. Triningsih, T. Utoro and Soeripto, 2014. Clinicopathological features of indonesian breast cancers with different molecular subtypes. Asian Pacific J. Cancer Prevent., 15: 6109-6113.

Wolfinger, M.T., W.A. Svrcek-Seiler, C. Flamm, I.L. Hofacker and P.F. Stadler, 2004. Efficient computation of RNA folding dynamics. J. Phys. A, 37: 4731-4741. DOI: 10.1088/0305-4470/37/17/005 